\title{
CrossCult: Empowering Reuse of Digital Cultural Heritage in Context-aware CrossCults of European History
}

\author{
Omar G. Bravo Quezada, Roberto A. García Vélez \\ GIHP4C Research Group, Systems Engineering Career \\ Salesian Polytechnic University \\ Cuenca, Ecuador \\ \{obravo,rgarciav\}@ups.edu.ec
}

\author{
Yolanda Blanco-Fernández, Martín López Nores \\ AtlantTIC Research Centre, \\ Dept. of Telematics Engineering, University of Vigo \\ Vigo, Spain \\ \{yolanda,mlnores\}@det.uvigo.es
}

\begin{abstract}
Human history is a mesh of interrelated facts that is normally presented in a simplistic way, which prevents from viewing the past as a shared, global experience. We build contextaware edutainment experiences that uncover associations among digital cultural resources to explain history in a holistic way.
\end{abstract}

Keywords-Semantic reasoning; interactivity; edutainment; History learning

\section{INTRODUCTION}

Even though Human history is a huge mess of interrelated facts and concepts, it is commonly presented in a simplistic and localistic way that promotes memorizing rather than understanding, disregarding cross-border aspects and preventing from viewing the past as a shared, global experience. This is especially noticeable in European history which by nature is highly interconnected- where most historyrelated experiences that are offered nowadays to the great public, from schools to museums, are siloed. To fight this situation, in this paper we describe the CrossCult system that uncovers implicit connections among digital cultural heritage resources that are somewhat associated, such as (i) a set of historic characters sharing birthplace or governing neighboring regions, (ii) events occurring concurrently or having a causeconsequence relationship, (iii) pieces of art with the same style or topic, and (iv) museums sharing part of a cross-culture collection, just to name a few examples. The goal is to exploit the huge amount of publicly available cultural repositories to assemble tailor-made interactive edutainment experiences for individuals and groups, with the goal of grasping history and helping appreciate the past in a holistic way.

For this purpose, our approach explores technologies borrowed from the field of the Semantic Web [1]. In particular, CrossCult requires a knowledge base where the history-related concepts and facts are semantically annotated, as well as semantic reasoning techniques aimed at inferring nexus among them. With the goal of promoting the entertainment in the History learning, CrossCult also exploits communication technologies and social networking functionalities to involve multiple venues in the resulting experiences. This way, for instance, users who are located in several cities/countries can compete each other through interactive games, which are orchestrated considering their spatio-temporal contexts, preferences, academic/professional trainings, etc. This results in a global cross-border approach that pursues a bottom-up reinterpretation of European (hi)stories which, to the best of our knowledge, has never been explored in literature.

This paper is organized as follows: Section II presents the architectural design of our system. The potential of CrossCult is illustrated through a sample scenario in Section III. Finally, Section IV concludes the paper.

\section{An Overview OF THE CROSSCUlt System}

As shown in Figure 1, the knowledge base of the CrossCult system has been developed as an OWL ontology [2], where the semantic annotations (metadata) of digital cultural heritage resources are formally specified, resulting into a network of interrelated concepts. Actually, these concepts are represented as class instances (nodes) and properties (edges) that describe historical characters, events, locations, as well as cultural assets and associated venues.

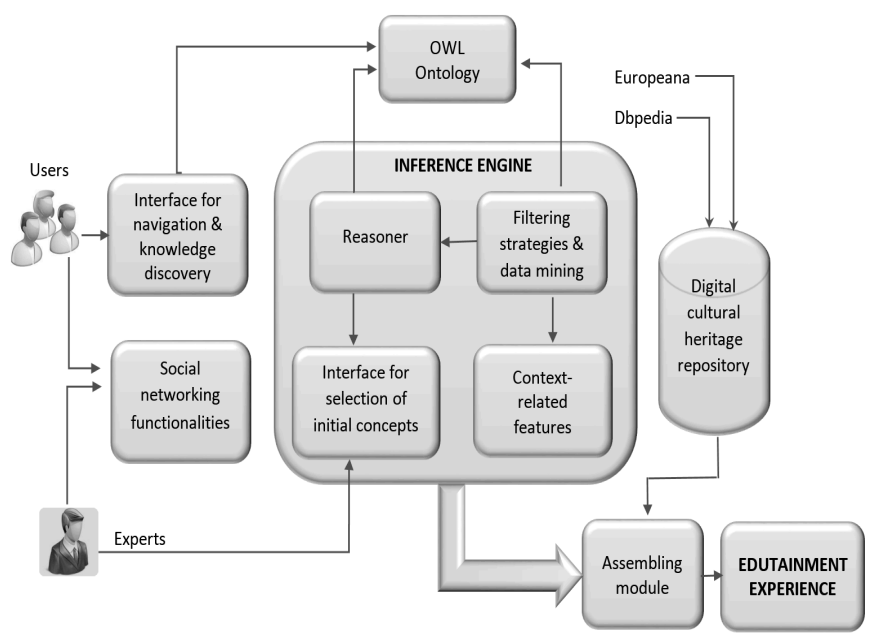

Figure 1. High-level architectural design of the CrossCult system

The CrossCult experiences are guided by experts (e.g. museum staff) who select an initial set of concepts from the 
OWL ontology, on which our edutainment experiences will be grounded. The system also supplies interfaces to the users in order to navigate through the OWL ontology, by exploring the metadata with the goal of discovering common facts among the expert-selected concepts. Likewise, the users are encouraged to share their discoveries with each other (both in the same venue as in remote venues) thanks to social networking functionalities.

The cornerstone of our system is the inference engine depicted in Figure 1, in which several tasks can be identified. On the one hand, this smart engine resorts to a reasoner to support the discovery of complex semantic relationships among the concepts formalized in the OWL ontology. In its development, we start from the semantic associations defined in [3], which identify relationships that capture both some kind of connectivity and similarity between the ontology concepts. Actually, our notion of similarity is not just a structural similarity, but a semantic similarity based on paths across the ontology where the concepts are involved. Following these paths, it is possible to detect, for example, that two nodes are similar if they have a common ancestor class (e.g. a Sculpture and a Painting are similar because both are instances belonging to the Creations class). Analogously, our reasoner is able to uncover a semantic association between two different Artists (e.g. a Painter and a Sculptor) who have made different Creations that are exhibited in a same Museum.

On the other hand, as depicted in Figure 1, our inference engine resorts to data mining techniques and (content-based and collaborative) filtering strategies [4] when it comes to binding together the knowledge discovered by the OWL reasoned and features related to the users' context (e.g. date, location, cognitive/emotional profiles, education, etc). The output of this process is finally considered by an assembling module that builds meaningful interactive edutainment experiences, by identifying and aggregating the multimedia contents and cultural resources -retrieved from publicly available datasets and repositories, such as DBPedia and Europeana- that best illustrate the semantic associations uncovered by the inference engine and the connections found by the users.

\section{A SAMPLE SCENARIO}

It is February 11th and several groups of high school students show up in several museums located in Madrid, Paris and Vilnius. Knowing the date and their locations, the CrossCult system proposes a competitive game in which the students have 5 minutes to discover common links between Gustavo Adolfo Bécquer (known to all Spanish students), Vincas Kudirka (known to all Lithuanians) and Fryderyk Chopin (known to everybody, but particularly present in French studies). Common facts joining the three people gain +3 points, and links for two of them gain +1 .

Using their smartphones, the students start navigating through a network of concepts in search for those common aspects, and their findings appear on common screens at the three venues: the three were male, they lived entirely in the XIX century, Bécquer and Kudirka were most known as poets, Kudirka and Chopin spent some time living in Warsaw,
Kudirka and Chopin, Kudirka and Chopin wrote some music... After 5 minutes, the search finishes and the points gained by participating grups are summed up. The French team wins!... but the most interesting fact joining the three teams was not discovered: they all died of tuberculosis. Tuberculosis was the topic chosen by the CrossCult system to drive the whole experience, starting from the fact that February 11th was designated by Pope John Paul II as "World Day of the Sick", and tuberculosis was found to be a common link for famous people from the three countries.

With the output provided by the inference engine of CrossCult, the staff of the three venues can collaboratively tell an interconnected (hi)story explaining what the date means, going briefly through the lives of Bécquer, Kudirka and Chopin and developing the topic of tuberculosis as a major cause of death throughout Europe during the XIX century. The (hi)story includes excerpts from famous pieces of art influenced by the disease: Puccini's opera "La Bohème", Verdi's "La Traviata", Dostoyevsky's book "Crime and punishment", Allan Poe's "The facts in the case of M. Valdemar", and recent movies like "Moulin Rouge!" and "The others", to illustrate the impact and the romantic lure of the disease during the XIX century.

\section{CONCLUSIONS}

The CrossCult system exploits the existing digital cultural heritage repositories in the development of new tailor-made interactive entertainment experiences for History learning. To this aim, we bring together the reasoning capabilities based on knowledge discovery from the Semantic Web, and technologies for content adaptation, context-awareness and personalization in mobile edutainment applications. The novel of the approach lies within the fact that we take advantage of the situational curiosity and serendipity to increase the retention of cross-border historical facts, gaining insight into the question of how the same facts may be interpreted differently from different social realities.

\section{ACKNOWLEDGMENT}

This work has been supported by the European Regional Development Fund (ERDF) and the Galician Regional Government under agreement for funding the AtlantTIC Research Center, as well as by the Ministerio de Educación y Ciencia (Gob. de España) research project TIN2013-42774-R.

\section{REFERENCES}

[1] T. Berners-Lee, J. Hendler and O. Lassila, "The Semantic Web," Scientific American, 2001.

[2] M. Dean, D. Connolly, F. Harmelen, J. Hendler, I. Horrocks, D. McGuinness, P. F. Patel-Schneider and L. Stein, "OWL Web Ontology Language 1.0 Reference," W3C Recommendation February 10th 2004. http://www.w3.org/TR/owl-ref/

[3] K. Anyanwu and A. Sheth, " $\rho$-Queries: enabling querying for Semantic Associations on the Semantic Web," in Proceedings of 12th International World Wide Web Conference (WWW), 2003, pp. 690-699.

[4] G. Adomavicius and A. Tuzhilin, "Towards the next generation of recommender systems: A survey of the state-of-the-art and possible extensions," IEEE Transaction on Knowledge and Data Engineering, vol. 17(6), pp. 734-749, June 2005. 